\title{
A REVIEW ON DERIVATIVE UV-SPECTROPHOTOMETRY ANALYSIS OF DRUGS IN PHARMACEUTICAL FORMULATIONS AND BIOLOGICAL SAMPLES REVIEW
}

\author{
VIVEKKUMAR K. REDASANI*, PRIYANKA R. PATEL, DIVYA Y. MARATHE, SURAJ R. CHAUDHARI, \\ ATUL A. SHIRKHEDKAR AND SANJAY J. SURANA
}

Department of Pharmaceutical Chemistry,

R. C. Patel Institute of Pharmaceutical Education and Research, Shirpur (MS), India

\begin{abstract}
The review article deals with theoretical aspects of Derivative UV-Spectrophotometry. The method gains significance using the first and second derivative of the transmission spectra with respect to wavelength. Generated optical derivatives are compared to the known numerical derivatives. The derivative spectra from $1^{\text {st }}$ to $4^{\text {th }}$ are consequently discussed. This provides valuable insight into the uses and limitations of this technique for chemical analysis. Measurement techniques and methods of obtaining derivative spectra are discussed. The degree of polynomial fit on the smoothness of derivative spectra and signal-to-noise ratio is described. Application of UV derivative spectrometry for determination of single and multicomponent analysis is shown. Derivative spectrophotometry possibly improves the selectivity and sensitivity of determination which has been illustrated.
\end{abstract}

Keywords: Derivative UV-Spectrophotometry, First Order Derivative spectra, Second Order Derivative spectra, Third Order Derivative spectra, Fourth Order Derivative spectra and area under curve.

\section{INTRODUCTION}

Derivative UV-spectrophotometry is an analytical technique of enormous implication commonly in obtaining mutually qualitative and quantitative in order from spectra that are of unresolved bands, with respect to qualitative and quantitative analysis, it uses first or higher derivatives of absorbance in accordance with wavelength [1]. Derivative spectroscopy was originally brought in 1950s with its applicability in a lot of features, but because of its complication in producing derivative spectra via UV-Visible spectroscopy the method found less practice. The weakness was conquering in 1970s with microcomputers which gave derivative spectra in more specific, simple, rapid and reproducible way. This made to enlarge applicability of derivative method; Derivatization of spectra augments selectivity by eradicates spectral interferences [2-3].

Derivative Spectroscopy

It is a spectroscopic technique that differentiates spectra's mainly in IR, UV-Visible absorption and Fluorescence spectrometry [4]. The objective with which derivative methods used in analytical chemistry are:

- $\quad$ Spectral differentiation

- $\quad$ Spectral resolution enhancement

- Quantitative analysis

Spectral differentiation

As a qualitative method that distinguish small variation between almost similar spectra's.

Spectral resolution enhancement

Overlapping spectral bands gets resolved to simply estimation the number of bands and their wavelengths.

\section{Quantitative analysis}

It facilitates multicomponent analysis and corrects the irrelevant background absorption. Derivative spectroscopy method forms the beginning of differentiation or resolution of overlapping bands; the vital characteristics of derivative process are that broad bands are suppressed relative to sharp bands [4].

\section{Measurement Techniques of the Derivative Spectroscopy}

Differentiation of a zero order spectrum of a combination of components shows the way to derivative spectrum of any order. There are many methods are used for discrimination of a spectrum viz., by analog or numeric method, spectral differentiation may be deliberate either graphically on paper or registered in a computer memory [5]. Measurement of derivative spectra value is achieved out by three methods viz. graphic measurement, numeric measurement, zero crossing technique

Graphic measurement

Graphic measurement is theoretical method for calculate the derivative spectra on paper, its manual method it suffer from disadvantage that it gives inaccurate results because the value can determined numerically can be abolish or diminish beyond restriction [5].

\section{Numeric measurement}

The method uses set of points where derivative values is carried out by estimating the derivative value at a given wavelength. It gives derivatives by spectral differentiation using suitable numerical algorithm [5].

Zero crossing technique

The method measures the derivative spectra at a particular wavelength, where the derivative crosses the point at zero line. Interference of one component in determination of other component can be eliminated by zero crossing technique [5].

Derivative Spectra

In quantitative analysis, derivative spectra enlarge difference between spectra to resolve overlapping bands [6]. The digital algorithm method called as Savitzky-Golay is most outstandingly referred for obtaining derivative spectra. In universal technique involves plotting the rate of change of the absorbance spectrum vs wavelength [7]. Derivative spectra can obtain by variety of experimental techniques; the differentiation can be done numerically even if the spectrum has been recorded digitally or in computerized readable form. When spectrum is scanned at a constant rate, real time derivative spectra can be recorded either by achieving the time derivative of the spectrum or by wavelength modulation [8]. Wavelength modulation device is used to record the derivative spectra, where a beam of radiation differs in wavelength by a small change (1-2 nm) and difference between the two readings is recorded, computerized method is widely used to obtain derivative curves.

Quantitatively for second or fourth order derivative curves, peak heights are measured of long-wave peak satellite or for short-wave peak satellite [9]. The degree of difficulty of derivative spectra increases with presence of satellite peaks. Second derivative spectra are represented by presence of two sharp peaks and troughs. The solvents have amazing effect over peaks [10]. On the basis of solvents polarity, peaks and troughs shifts either to shorter or longer wavelength (Fig. 1).

The way of obtaining the derivative orders

Derivative spectroscopy accomplishes conversion of a normal or zero order spectrums to its first, second or higher derivative spectrum. It yields considerable changes in shape of derivative achieved. Appropriate selection of derivative order gives useful separation of overlapped signals. Criterion like signals height, their width and distance between maxima in basic spectrum is achieved by optimal derivative order, to attain wide spectrum bands it is expected to use low orders and for narrow spectral bands-higher orders. A Gaussian band represents an ideal absorption band gives clear idea about transformation occurring in the derivative spectra. Plotting absorbance versus wavelength gives a graph, showing peak with maxima and minima (also points of inflection) that is supposed to passed through zero on the ordinate [10] (Fig. 2). 


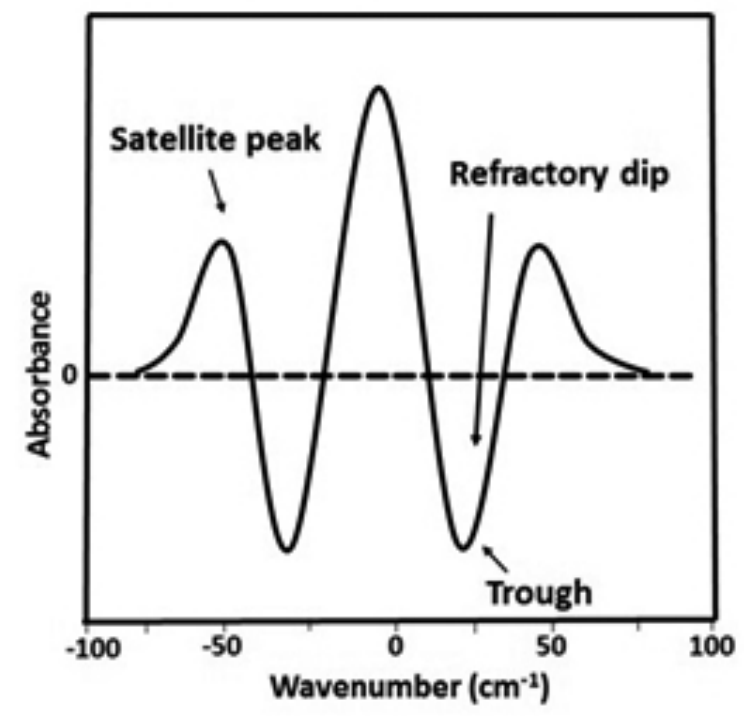

Figure 1: Derivative Spectra.
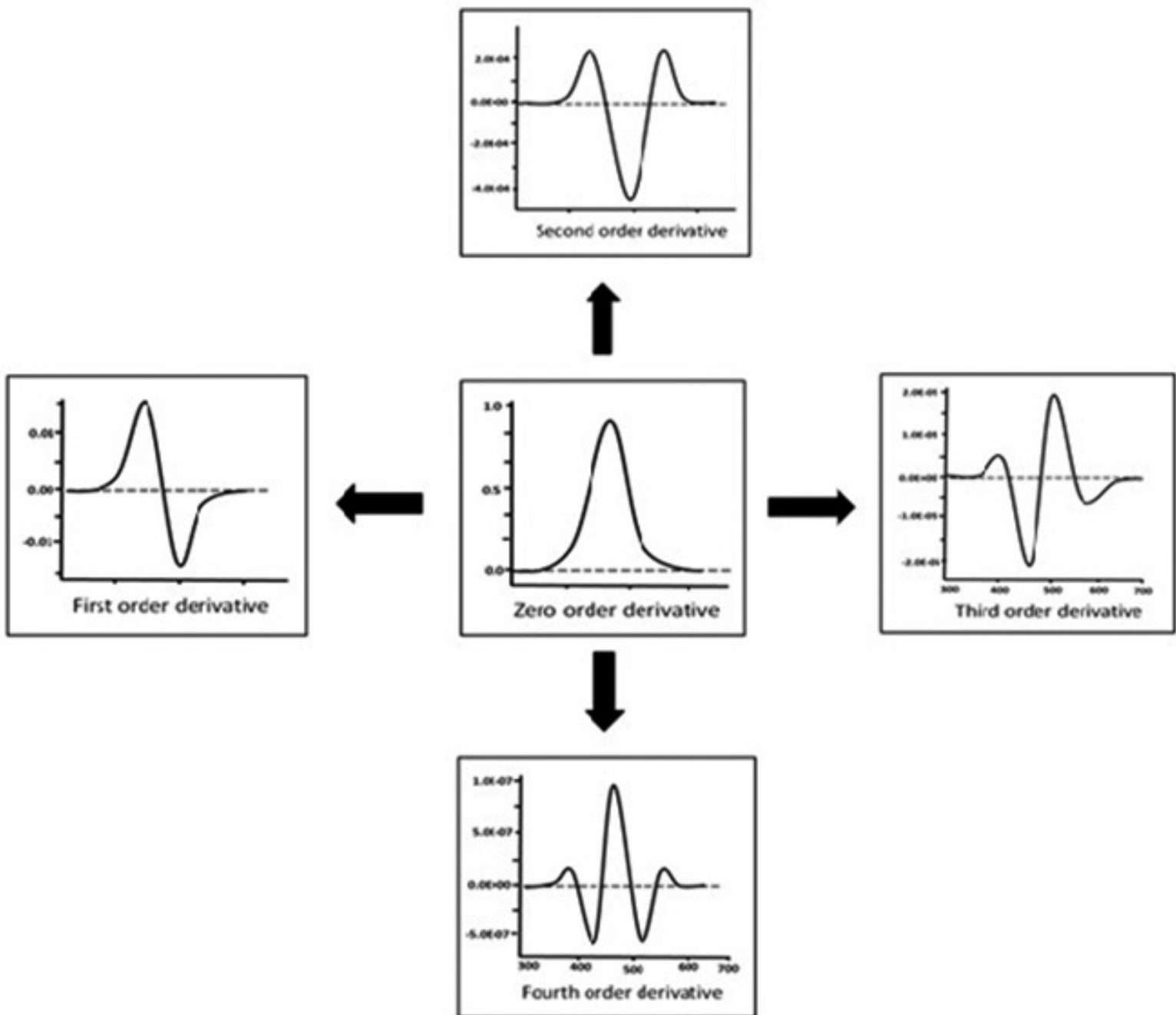

Figure 2: Oder of Derivative Spectra. 


\section{Zero order derivative spectrum}

Zero order derivative is initial step of giving further derivatives i.e., zero $^{\text {th }}$ order spectrum can give $\mathrm{n}^{\text {th }}$ order derivative. In derivative spectroscopy, $\mathrm{D}^{0}$ spectrum i.e. zeroth order is a representative feature of normal absorption spectrum [12]. The $1^{\text {st }}, 2^{\text {nd }}, 3^{\text {rd }}$ and $4^{\text {th }}$ order derivative spectra can be obtained directly from the zero ${ }^{\text {th }}$ order spectrum. An increase in order of derivatives increases the sensitivity of determination [14]. If a spectrum is expressed as absorbance (A) as a function of wavelength $(\lambda)$, the derivative spectra is given as,

\section{$A=f(\lambda)$,}

\section{First order derivative spectrum}

Spectra obtained by derivatizing zero order spectrum once. It is a plot of change of absorbance with wavelength against wavelength ${ }^{10}$ i.e. rate of change of the absorbance with wavelength,

$\mathrm{dA} / \mathrm{d} \lambda=\mathrm{f}^{\prime}(\lambda)$

Even if in derivatized form it is more complex than zero order spectrum. First order spectra passes through zero as $\lambda$ max of the absorbance band. Absorbance band of first order derivative shows certain positive and negative band with maxima and minima [6]. By scanning the spectrum with a minimum and constant difference between two wavelengths, dual-wavelength spectrophotometer obtains first-derivative spectra [8]

Second order derivative spectrum

Derivatizing the absorbance spectrum twice gives this type of spectra [7].

It is a plot of curvature of absorption spectrum against wavelength [16].

$\mathrm{d}^{2} \mathrm{~A} / \mathrm{d} \lambda^{2}=\mathrm{f}^{\prime \prime}(\lambda)$

Second derivative has direct relation with concentration i.e. directly proportional. $\mathrm{d}^{2} \mathrm{~A} / \mathrm{d} \lambda^{2}$ must be large, large the ratio greater is the sensitivity [8] The method is useful in obtaining atomic and gas molecular spectra.

Third order derivative spectrum

Unlike second order spectrum third derivative spectrum shows disperse function to that of original curve [11].

$\mathrm{d}^{3} \mathrm{~A} / \mathrm{d} \lambda^{3}=\mathrm{f} " '(\lambda)$

Fourth-derivative spectrum

Fourth order is inverted spectrum of second order and has a sharper centra peak than the original band, Narrow bands are selectively determined by fourth derivative (UV-high pressure) [9].

$$
\mathrm{d}^{4} \mathrm{~A} / \mathrm{d} \lambda^{4}=\mathrm{f}^{\prime}(\lambda)
$$

\section{Polynomial degree}

Polynomial degree has a great impact on number of polynomial points rather than on shape of derivative [5]. The scope of polynomial is less; differentiation of spectra of half-width is used by low degree polynomials and that for spectra of small half -width by higher degree polynomials [5]. Distorted derivative spectrum is a result of inappropriate polynomial degree In case of multicomponent analysis, the spectral differences of assayed compounds and their selective determination can be increased by the use of different polynomial degrees [2].

\section{Signal-to-noise ratio}

Derivative technique becomes difficult when used with higher orders that produce signal-to-noise worse [1]. The result is decrease in $\mathrm{S} / \mathrm{N}$ with highe orders. The noise is responsible for sharpest features in the spectrum. There are increased demands on low-noise characteristics of the spectrophotometer by negative effect of derivatization on $\mathrm{S} / \mathrm{N} .{ }^{5} \mathrm{~S} / \mathrm{N}$ can be improved prior to derivatization if spectrophotometer would scan spectra and average multiple spectra [6]. Best signal-to-noise ratio can be obtained by taking the difference between the highest maximum and the lowest minimum, but this leads to enhanced sensitivity to interference from other components [2]. Noise of signal is expressed by standard deviation $\sigma$. Standard deviation $\sigma_{0}$ expresses the noise of normal spectrum of the absorbance of blank while standard deviation $\sigma_{n}$ expresses $\mathrm{n}^{\text {th }}$ order derivative that can be calculated by $\sigma_{0}[1,2]$.

\section{Smoothing of spectra}

Increase in signal-to-noise ratio generates many worse conditions, to lessen the condition or to decrease the high-frequency noise, technique is used viz; low-pass filtering or smoothing. Smoothing is an operation that is performed on spectra separately on each row of the data and acts on adjacent variables [14]. The noise can be lower significantly without loss of the signal of interest when variables are close to each other in the data matrix and contain similar information [12]. Derivative spectrum may be altered with a high degree of smoothing so, care must be taken $[1,6]$. The smoothing effect depends upon two variables mainly on: (a) Frequency of smoothing and (b) the smoothing ratio i.e. ratio of width of the smoothed peak to the number $\mathrm{M}$ of data points [15].
Advantages and Disadvantages of Derivative UV-Spectrophotometry Advantages

UV Derivative Spectroscopy has increased sensitivity and selectivity. It has multiple advantages viz., single component analysis and simultaneous determination of several components in a mixture, determination of traces in matrix, protein and amino acid analysis, environmental analysis, identification of organic and inorganic compounds [5].

Specific benefits of derivative spectral analysis includes viz;

- Even in small wavelength range, in presence of two or more overlapped peaks, absorbance bands can be identified.

- In presence of strong and sharp absorbance peak, weak and small absorbance peak can be identified.

- Broad absorbance spectrum gives clear idea about the particular wavelength at that maximum spectrum.

- $\quad$ Even in presence of existed background absorption, the quantitative analysis can studied as there is linear relationship between the derivative values and the concentration levels $[13,14]$

\section{Disadvantages}

Even though it is sensitive method still it is highly susceptible to various parameters. The method is limited to particular system only and has limited applications due to its less reproducibility. The method is second choice when existing instrumental method (which measures signal) is absent. It is less accurate in measuring zero-crossing spectra. There is likeness in shape of derivative spectra and zero order spectrum, so small variation in a basic spectrum can strongly modify derivative spectrum. Poor reproducibility can alter results in way when different spectrophotometers used for zero order spectra gives similar results but derivatization of them display different [15].

\section{Applications}

a. Single component analysis: Derivative spectrophotometry analyses single component (Table 1) along with Area under Curve (Table 3) in pharmaceutical formulation.

b. Multicomponent analysis: Derivative spectrophotometry in pharmaceutical analysis analyses more than one component in presence of other components i.e. simultaneous determination of two or more compounds. Spectral derivatization can remove the prevalence caused by spectra of disturbing compounds (Table 2) [3]

c. Bioanalytical application: Besides pharmaceutical analysis, derivative spectrophotometry may be applied to different areas. Determination of compounds in various biological samples like plasma, serum, urine and brain tissue [2]. Amphotericine [52] and Diazepam [26] has been determined in human plasma with its order of derivatives.

d. Forensic toxicology: Derivative spectroscopy has its application in toxicology especially of illicit drugs viz; amphetamine, ephedrine, meperidine, diazepam, etc. and can also be used in mixtures [1].

e. Trace analysis: Derivative signal processing technique is widely used in practical analytical work in measurement of small amounts of substances in the presence of large amounts of potentially interfering substances [4]. Due to such interference, analytical signals becomes weak, noisy and superimposed on large background signals. The conditions like non-specific broadband interfering absorption, non-reproducible cuvette positioning, dirt or fingerprints on the cuvette walls, imperfect cuvette transmission matching, and solution turbidity results in degraded measurement precision is by sample-tosample baseline shifts [4]. Baseline shifts may be due to practical errors, either are weak wavelength dependence (small particle turbidity) or wavelengthindependent (light blockage caused by bubbles or large suspended particles). So, there is need of differentiation of relevant absorption from these sources of baseline shift [5]. It is expected to suppress broad background by differentiation with a aim that it reduces variations in background amplitude from sample-tosample. This results in improved precision and measurement in many instances, especially in case if there is a lot of uncontrolled variability in the background and when the analyte signal is small compared to the background [4].

\section{CONCLUSION}

Derivative Spectrophotometry is presently available with software's controlling modern spectrophotometers. This makes easy to analyst in obtaining useful information from spectra of respective compounds. The derivatives of UV spectra give applicable information in elucidating compounds in pharmaceutical formulation. This present article provides complete understanding about derivative spectrophotometry technique \& its applications. 
Table 1: Single Component determination of analyte in Pharmaceutical sample.

\begin{tabular}{|c|c|c|c|c|c|}
\hline Drug & Order of derivative & Wavelength selected(nm) & $\begin{array}{c}\text { Linearity } \\
(\mu \mathrm{g} / \mathrm{ml})\end{array}$ & Year of publication & Reference \\
\hline Efavirenz & $\begin{array}{l}\mathrm{D}_{0} \\
\mathrm{D}_{1}\end{array}$ & $\begin{array}{l}239 \mathrm{~nm} \\
248 \mathrm{~nm}\end{array}$ & $5-40$ & 2014 & 16 \\
\hline Carbimazole & $\begin{array}{l}\mathrm{D}_{1} \\
\mathrm{D}_{2} \\
\mathrm{D}_{3} \\
\mathrm{D}_{4}\end{array}$ & $\begin{array}{l}314 \mathrm{~nm} \\
300 \mathrm{~nm} \\
289 \mathrm{~nm} \\
320 \mathrm{~nm}\end{array}$ & $2-18$ & 2014 & 17 \\
\hline Aripiprazole & $\mathrm{D}_{0}$ & $217 \mathrm{~nm}$ & $1-6$ & 2014 & 18 \\
\hline Chlorthalidone & $\begin{array}{l}\mathrm{D}_{1} \\
\mathrm{D}_{2}\end{array}$ & $\begin{array}{l}278 \mathrm{~nm} \& 288 \mathrm{~nm} \\
286 \mathrm{~nm} \& 292 \mathrm{~nm}\end{array}$ & $1-25$ & 2014 & 19 \\
\hline Famotidine & $\begin{array}{l}\mathrm{D}_{1} \\
\mathrm{D}_{2}\end{array}$ & $\begin{array}{c}\text { Valley-272.2nm } \\
\text { Max.amplitude-287.7nm }\end{array}$ & $4-12$ & 2014 & 20 \\
\hline Lacosamide & $\mathrm{D}_{1}$ & $250 \mathrm{~nm}$ & $5-50$ & 2013 & 21 \\
\hline Dronedarne & $\begin{array}{l}\mathrm{D}_{0} \\
\mathrm{D}_{1}\end{array}$ & $\begin{array}{l}290 \mathrm{~nm} \\
275 \mathrm{~nm}\end{array}$ & $4-20 n$ & 2013 & 23 \\
\hline Irbesartan & $\begin{array}{l}\mathrm{D}_{3} \\
\mathrm{D}_{4}\end{array}$ & $\begin{array}{l}224 \mathrm{~nm} \\
230 \mathrm{~nm}\end{array}$ & $\begin{array}{l}2-20 \\
2-14\end{array}$ & 2012 & 24 \\
\hline Ciprofibrate & $\mathrm{D}_{1}$ & $232 \mathrm{~nm}$ & $2-12$ & 2012 & 25 \\
\hline Diazepam & $\mathrm{D}_{4}$ & $306-333 \mathrm{~nm}$ & $2-10$ & 2012 & 26 \\
\hline Stavudine & $\begin{array}{l}\mathrm{D}_{0} \\
\mathrm{D}_{1} \\
\mathrm{D}_{2}\end{array}$ & $\begin{array}{c}265 \mathrm{~nm} \\
250.8 \mathrm{~nm} \\
232.8 \mathrm{~nm} \\
\end{array}$ & $\begin{array}{l}2-20 \\
2-20 \\
2-20\end{array}$ & 2012 & 27 \\
\hline Diarcerein & $\mathrm{D}_{1}$ & $259.4 \& 274.2 \mathrm{~nm}$ & $2-12$ & 2012 & 28 \\
\hline Neomycin & $\mathrm{D}_{1}$ & $277 \mathrm{~nm}$ & $0.10-0.51$ & 2011 & 29 \\
\hline Fluconazole & $\mathrm{D}_{1}$ & $268 \mathrm{~nm}$ & $150-350$ & 2011 & 30 \\
\hline Ranitidine $\mathrm{HCl}$ & $\begin{array}{l}\mathrm{D}_{0} \\
\mathrm{D}_{1}\end{array}$ & $\begin{array}{l}312 \mathrm{~nm} \\
332 \mathrm{~nm}\end{array}$ & $0.5-35.1$ & 2011 & 32 \\
\hline Ritonavir & $\mathrm{D}_{2}$ & $232 \mathrm{~nm}$ & $10-50$ & 2011 & 33 \\
\hline Alprazolam & $\begin{array}{c}\text { Overlain derivative } \\
\text { spectroscopy }\end{array}$ & $521 \mathrm{~nm}$ & $5-45$ & 2011 & 34 \\
\hline Tropicamide & $\begin{array}{l}\mathrm{D}_{3} \\
\mathrm{D}_{4}\end{array}$ & $\begin{array}{l}263.8 \mathrm{~nm} \\
255.4 \mathrm{~nm}\end{array}$ & $\begin{array}{l}10-100 \\
10-100 \\
\end{array}$ & 2010 & 2 \\
\hline Olanzapine & $\begin{array}{l}\mathrm{D}_{1} \\
\mathrm{D}_{2}\end{array}$ & $\begin{array}{l}222 \mathrm{~nm} \\
230 \mathrm{~nm}\end{array}$ & $\begin{array}{l}2-10 \\
2-10\end{array}$ & 2010 & 2 \\
\hline Galanthamine & $\begin{array}{l}\mathrm{D}_{1} \text { zero crossing } \\
\text { spectroscopy }\end{array}$ & $277.4 \mathrm{~nm}$ & $30-80$ & 2010 & 2 \\
\hline Cisapride & $\begin{array}{l}\mathrm{D}_{1} \\
\mathrm{D}_{2} \\
\end{array}$ & $\begin{array}{l}264,300 \mathrm{~nm} \\
276,290 \mathrm{~nm}\end{array}$ & $\begin{array}{l}2-10 \\
2-10 \\
\end{array}$ & 2010 & 35 \\
\hline Cefuroxime axetil & $\mathrm{D}_{1}$ & $281 \mathrm{~nm}$ & $4-30$ & 2010 & 36 \\
\hline Gemifloxacin mesylate & $\begin{array}{l}\mathrm{D}_{0} \\
\mathrm{D}_{1} \\
\mathrm{D}_{2}\end{array}$ & $\begin{array}{l}430 \mathrm{~nm} \\
480 \mathrm{~nm} \\
500 \mathrm{~nm}\end{array}$ & $\begin{array}{l}2-14 \\
1-10 \\
1-15\end{array}$ & 2010 & 37 \\
\hline Letrozole & $\begin{array}{l}\mathrm{D}_{0} \\
\mathrm{D}_{1} \\
\mathrm{D}_{2}\end{array}$ & $\begin{array}{l}240 \mathrm{~nm} \\
224 \mathrm{~nm} \\
241 \mathrm{~nm}\end{array}$ & $0.25-20$ & 2010 & 38 \\
\hline Losartan potassium & $\begin{array}{l}\mathrm{D}_{0} \\
\mathrm{D}_{1}\end{array}$ & $\begin{array}{l}205 \mathrm{~nm} \\
234 \mathrm{~nm}\end{array}$ & $\begin{array}{c}3-7 \\
4-16 \\
\end{array}$ & 2010 & 39 \\
\hline Lopinavir & $\mathrm{D}_{1}$ & $220 \mathrm{~nm}$ & $5-35$ & 2010 & 40 \\
\hline Ropinirole & $\mathrm{D}_{1}$ & $262.5 \mathrm{~nm}$ & $4-20$ & 2010 & 41 \\
\hline
\end{tabular}


J. Chil. Chem. Soc., 63, № 3 (2018)

\begin{tabular}{|c|c|c|c|c|c|}
\hline Metoprolol & $\begin{array}{l}D_{0} \\
D_{1} \\
D_{2} \\
D_{3}\end{array}$ & $\begin{array}{c}276 \mathrm{~nm} \\
265,278,285 \mathrm{~nm} \\
276,279,287,282 \mathrm{~nm} \\
275,278,218 \mathrm{~nm}\end{array}$ & $5-15$ & 2010 & 42 \\
\hline Venlafaxine $\mathrm{HCl}$ & $\mathrm{D}_{3}$ & $274 \mathrm{~nm}$ & $40-120$ & 2010 & 43 \\
\hline Sertraline $\mathrm{HCl}$ & $\mathrm{D}_{1}$ & $475.72-588.40 \mathrm{~nm}$ & $5-100$ & 2009 & 2 \\
\hline Estapenem & $\begin{array}{l}D_{1} \\
D_{2}\end{array}$ & $\begin{array}{c}316 \mathrm{~nm} \\
298 \mathrm{~nm} \& 316 \mathrm{~nm}\end{array}$ & $\begin{array}{l}4-60 \\
2-28\end{array}$ & 2009 & 2 \\
\hline Candesartan cilexetil & $\mathrm{D}_{1}$ & $270.1 \mathrm{~nm}$ & $6-32$ & 2009 & 44 \\
\hline Gentamicin sulfate & $\mathrm{D}_{3}$ & $281 \mathrm{~nm}$ & $0.004-0.008 \%$ & 2009 & 45 \\
\hline Pioglitazone & $\begin{array}{l}\mathrm{D}_{0} \\
\mathrm{D}_{2}\end{array}$ & $\begin{array}{c}270 \mathrm{~nm} \\
272-287.4 \mathrm{~nm}\end{array}$ & $\begin{array}{l}5-20 \\
2-12\end{array}$ & 2009 & 46 \\
\hline Benazepril & $\begin{array}{l}\mathrm{D}_{1} \\
\mathrm{D}_{2} \\
\mathrm{D}_{3}\end{array}$ & $\begin{array}{l}213 \mathrm{~nm} \\
219 \mathrm{~nm} \\
223 \mathrm{~nm}\end{array}$ & $1.2-12$ & 2009 & 47 \\
\hline Ezetimibe & $\begin{array}{l}\mathrm{D}_{1} \\
\mathrm{D}_{2} \\
\mathrm{D}_{3}\end{array}$ & $\begin{array}{c}259.5 \mathrm{~nm} \\
269 \mathrm{~nm} \\
248 \mathrm{~nm}\end{array}$ & $\begin{array}{l}4-14 \\
4-14 \\
4-16\end{array}$ & 2008 & 48 \\
\hline Drotaverine & $\mathrm{D}_{2}$ & $247.4 \mathrm{~nm}$ & $4-32$ & 2008 & 49 \\
\hline Tenofovir & $\begin{array}{l}\mathrm{D}_{0} \\
\mathrm{D}_{1}\end{array}$ & $\begin{array}{l}260 \mathrm{~nm} \\
273 \mathrm{~nm}\end{array}$ & $5-40$ & 2008 & 50 \\
\hline Prednisolone & $\mathrm{D}_{0}$ & $242 \mathrm{~nm}$ & $0.36-50.46$ & 2008 & 51 \\
\hline Amphotericine & $\mathrm{D}_{0}$ & $300 \mathrm{~nm} \& 500 \mathrm{~nm}$ & $1.25-5$ & 2008 & 52 \\
\hline Amoxicillin & $\begin{array}{l}\mathrm{D}_{1} \\
\mathrm{D}_{2}\end{array}$ & $\begin{array}{l}255.8 \mathrm{~nm} \\
249.2 \mathrm{~nm}\end{array}$ & $3.2-48$ & 2008 & 53 \\
\hline Losartan & $\mathrm{D}_{1}$ & $220-320 \mathrm{~nm}$ & $2-50$ & 2004 & 55 \\
\hline
\end{tabular}

Table 2: Simultaneous determination of two or more compounds in Pharmaceutical sample.

\begin{tabular}{|c|c|c|c|c|c|}
\hline Drug & Order of derivative & Wavelength (nm) & Linearity $(\mu \mathrm{g} / \mathrm{ml})$ & Year & Reference \\
\hline $\begin{array}{l}\text { 17- } \beta \text { Estradiol \& } \\
\text { Drospirenone }\end{array}$ & $\begin{array}{c}\mathrm{D}_{1} \\
\text { Zero crossing }\end{array}$ & $\begin{array}{l}208 \mathrm{~nm} \\
282 \mathrm{~nm}\end{array}$ & $\begin{array}{c}0.5-8 \\
0.5-32\end{array}$ & 2015 & 56 \\
\hline $\begin{array}{c}\text { Tramadol HCl\& } \\
\text { Paracetamol }\end{array}$ & $\mathrm{D}_{1}$ & $200-500 \mathrm{~nm}$ & $\begin{array}{c}6-48 \\
25-112\end{array}$ & 2015 & 57 \\
\hline $\begin{array}{l}\text { Acetaminophen, } \\
\text { Diphenhydramine \& } \\
\text { pseudoephedrine }\end{array}$ & $\begin{array}{l}\text { Zero crossing } \\
\text { method }\end{array}$ & $\begin{array}{l}281.5 \mathrm{~nm} \\
226 \mathrm{~nm} \\
218 \mathrm{~nm}\end{array}$ & $\begin{array}{c}5-50 \\
0.25-4 \\
0.5-5\end{array}$ & 2015 & 58 \\
\hline $\begin{array}{l}\text { Chloramphenicol, } \\
\text { Dexamethasone \& } \\
\text { Naphazoline }\end{array}$ & $\mathrm{D}_{1}$ & $220 \mathrm{~nm}$ & $\begin{array}{c}20-70 \\
6-14 \\
3-8\end{array}$ & 2015 & 59 \\
\hline $\begin{array}{l}\text { Zofenopril \& } \\
\text { Fluvastatin }\end{array}$ & $\begin{array}{l}\mathrm{D}_{1} \\
\mathrm{D}_{2} \\
\mathrm{D}_{3} \\
\mathrm{D}_{1} \\
\mathrm{D}_{2} \\
\mathrm{D}_{3}\end{array}$ & $\begin{array}{l}270.85 \mathrm{~nm} \\
286.38 \mathrm{~nm} \\
253.90 \mathrm{~nm} \\
339.03 \mathrm{~nm} \\
252.57 \mathrm{~nm} \\
258.50 \mathrm{~nm}\end{array}$ & $7.65-22.94$ & 2015 & 60 \\
\hline $\begin{array}{l}\text { Nebivolol \& } \\
\text { Clinidipine }\end{array}$ & $\mathrm{D}_{1}$ & $\begin{array}{l}221.6 \mathrm{~nm} \\
249 \mathrm{~nm}\end{array}$ & $\begin{array}{l}4-20 \\
5-25\end{array}$ & 2012 & 61 \\
\hline $\begin{array}{l}\text { Ibuprofen \& } \\
\text { Paracetamol }\end{array}$ & $\mathrm{D}_{1}$ & $200-235 \mathrm{~nm}$ & $\begin{array}{l}12-32 \\
20-40\end{array}$ & 2014 & 62 \\
\hline $\begin{array}{l}\text { Levofloxacin hemihydrate } \\
\qquad \& \\
\text { Ambroxol hydrochloride }\end{array}$ & $\begin{array}{c}\mathrm{D}_{1} \\
\text { Zero crossing }\end{array}$ & $\begin{array}{l}255.70 \mathrm{~nm} \\
253 \mathrm{~nm}\end{array}$ & $\begin{array}{c}5-40 \\
3-10.5\end{array}$ & 2014 & 63 \\
\hline $\begin{array}{l}\text { Gatifloxacin \& } \\
\text { Prednisolone }\end{array}$ & $\mathrm{D}_{1}$ & $\begin{array}{l}348 \mathrm{~nm} \\
263 \mathrm{~nm}\end{array}$ & $\begin{array}{l}3-21 \\
6-42\end{array}$ & 2014 & 64 \\
\hline
\end{tabular}


J. Chil. Chem. Soc., 63, № 3 (2018)

\begin{tabular}{|c|c|c|c|c|c|}
\hline $\begin{array}{l}\text { Diclofenac potassium, } \\
\text { Paracetamol \& } \\
\text { Serratiopeptidase }\end{array}$ & $\mathrm{D}_{1}$ & $\begin{array}{l}252 \mathrm{~nm} \\
276 \mathrm{~nm} \\
330 \mathrm{~nm}\end{array}$ & $\begin{array}{l}2-15 \\
2-30 \\
2-80\end{array}$ & 2014 & 65 \\
\hline $\begin{array}{l}\text { Rosuvastatin calcium \& } \\
\text { Fenofibrate }\end{array}$ & Zero crossing point & $\begin{array}{l}224.11 \mathrm{~nm} \\
243.29 \mathrm{~nm}\end{array}$ & $\begin{array}{c}16-48 \\
4-12\end{array}$ & 2013 & 66 \\
\hline $\begin{array}{l}\text { Paracetamol \& } \\
\text { Etodolac }\end{array}$ & Zero crossing point & $\begin{array}{l}247 \mathrm{~nm} \\
280 \mathrm{~nm}\end{array}$ & $\begin{array}{l}5-25 \\
2-18\end{array}$ & 2013 & 67 \\
\hline $\begin{array}{l}\text { Salbutamol sulphate \& } \\
\text { Ketotifen fumarate }\end{array}$ & $\begin{array}{l}\mathrm{D}_{1} \\
\mathrm{D}_{1}\end{array}$ & $\begin{array}{l}257 \mathrm{~nm} \\
278 \mathrm{~nm}\end{array}$ & $\begin{array}{l}5-45 \\
5-35\end{array}$ & 2013 & 68 \\
\hline $\begin{array}{l}\text { Pioglitazone } \mathrm{HCl} \& \\
\text { Glimepride }\end{array}$ & $\begin{array}{c}\mathrm{D}_{1} \\
\text { Zero crossing }\end{array}$ & $\begin{array}{l}225 \mathrm{~nm} \\
248 \mathrm{~nm}\end{array}$ & $\begin{array}{l}5-30 \\
4-20\end{array}$ & 2013 & 69 \\
\hline $\begin{array}{l}\text { Levocetrizine } \mathrm{HCl} \& \\
\text { Phenylephrine } \mathrm{HCl}\end{array}$ & $\mathrm{D}_{0}$ & $\begin{array}{l}230 \mathrm{~nm} \\
216 \mathrm{~nm}\end{array}$ & $\begin{array}{c}3-9 \\
6-18\end{array}$ & 2013 & 70 \\
\hline $\begin{array}{l}\text { Ofloxacin \& } \\
\text { Ornidazole }\end{array}$ & $\mathrm{D}_{1}$ & $\begin{array}{c}278 \mathrm{~nm} \\
293.6 \mathrm{~nm}\end{array}$ & $\begin{array}{c}0.5-10 \\
2-30\end{array}$ & 2013 & 71 \\
\hline $\begin{array}{c}\text { Tolperisone \& } \\
\text { Paracetamol }\end{array}$ & $\mathrm{D}_{1}$ & $\begin{array}{l}261 \mathrm{~nm} \\
243 \mathrm{~nm}\end{array}$ & $\begin{array}{c}0-2.5 \\
3-9\end{array}$ & 2013 & 72 \\
\hline $\begin{array}{l}\text { Paracetamol \& } \\
\text { Domperidone }\end{array}$ & $\mathrm{D}_{1}$ & $\begin{array}{l}250 \mathrm{~nm} \\
285 \mathrm{~nm}\end{array}$ & $\begin{array}{l}5-25 \\
0.8-5\end{array}$ & 2013 & 73 \\
\hline $\begin{array}{l}\text { Drotaverine \& } \\
\text { Mefenemic acid }\end{array}$ & $\mathrm{D}_{1}$ & $\begin{array}{c}253.8 \mathrm{~nm} \\
304 \mathrm{~nm}\end{array}$ & $4-24$ & 2013 & 74 \\
\hline $\begin{array}{l}\text { Moxifloxacin \& } \\
\text { Cefixime }\end{array}$ & $\begin{array}{l}1^{\text {st }} \text { zero crossing } \\
\text { wavelength }\end{array}$ & $\begin{array}{c}200-400 \mathrm{~nm} \\
287 \mathrm{~nm} \& 317.9 \mathrm{~nm}\end{array}$ & $\begin{array}{l}1-16 \\
1-15\end{array}$ & 2012 & 75 \\
\hline $\begin{array}{l}\text { Ibuprofen \& } \\
\text { Famotidine }\end{array}$ & $\mathrm{D}_{1}$ & $\begin{array}{c}249 \mathrm{~nm} \\
263.6 \mathrm{~nm}\end{array}$ & $\begin{array}{c}4-20 \\
120-600\end{array}$ & 2012 & 76 \\
\hline $\begin{array}{l}\text { Telmisartan \& } \\
\text { Metoprolol }\end{array}$ & $\mathrm{D}_{2}$ & $\begin{array}{c}299.5 \mathrm{~nm} \\
224 \mathrm{~nm}\end{array}$ & 3-15 & 2012 & 77 \\
\hline $\begin{array}{l}\text { Nebivolol \& } \\
\text { S-Amlodipine }\end{array}$ & $\begin{array}{l}\mathrm{D}_{0} \\
\mathrm{D}_{1}\end{array}$ & $\begin{array}{c}280 \& 364 \mathrm{~nm} \\
294 \& 279.7 \mathrm{~nm}\end{array}$ & $\begin{array}{c}10-60 \\
5-30\end{array}$ & 2012 & 78 \\
\hline $\begin{array}{l}\text { Lamivudine \& } \\
\text { Zidovudine }\end{array}$ & $\mathrm{D}_{1}$ & $\begin{array}{l}279 \mathrm{~nm} \\
300 \mathrm{~nm}\end{array}$ & $10-50$ & 2012 & 79 \\
\hline $\begin{array}{l}\text { Ondansetron \& } \\
\text { Pantoprazole }\end{array}$ & $\mathrm{D}_{1}$ & $\begin{array}{c}288.5 \mathrm{~nm} \\
310 \mathrm{~nm}\end{array}$ & $\begin{array}{c}0.5-25 \\
5-25\end{array}$ & 2012 & 80 \\
\hline $\begin{array}{l}\text { Drotaverine \& } \\
\text { Nimesulide }\end{array}$ & $\begin{array}{l}\text { Ratio derivative } \\
\text { spectroscopy }\end{array}$ & $\begin{array}{c}254 \& 274.68 \mathrm{~nm} \\
221.09 \& 232.067 \mathrm{~nm}\end{array}$ & $\begin{array}{c}8-24 \\
20-60\end{array}$ & 2012 & 81 \\
\hline $\begin{array}{l}\text { Metoprolol \& } \\
\text { Amlodipine }\end{array}$ & $\begin{array}{c}\text { Ratio derivative } \\
\text { spectroscopy } \\
\mathrm{D}_{1} \\
\end{array}$ & $\begin{array}{l}277.01 \mathrm{~nm} \\
235.62 \mathrm{~nm}\end{array}$ & $\begin{array}{l}50-250 \\
5-25\end{array}$ & 2012 & 82 \\
\hline $\begin{array}{l}\text { Aceclofenac \& } \\
\text { Tizanidine }\end{array}$ & $\begin{array}{l}1^{\text {st }} \text { by zero crossing } \\
\text { method }\end{array}$ & $\begin{array}{l}250 \mathrm{~nm} \\
313 \mathrm{~nm}\end{array}$ & $\begin{array}{l}2-20 \\
1-10\end{array}$ & 2011 & 83 \\
\hline $\begin{array}{c}\text { Atrovastatin calcium \& } \\
\text { Amlodipine }\end{array}$ & $\begin{array}{l}D_{0} \\
D_{1}\end{array}$ & $\begin{array}{l}241 \mathrm{~nm} \\
250 \mathrm{~nm}\end{array}$ & $\begin{array}{c}0-14 \\
0-7\end{array}$ & 2011 & 84 \\
\hline $\begin{array}{c}\text { Gemifloxacin mesylate \& } \\
\text { Ambroxol } \mathrm{HCl}\end{array}$ & $\mathrm{D}_{1}$ & $\begin{array}{c}272 \mathrm{~nm} \\
249.5 \mathrm{~nm}\end{array}$ & $\begin{array}{l}8-40 \\
6-30\end{array}$ & 2011 & 85 \\
\hline $\begin{array}{c}\text { Tenofovir disoproxil } \\
\text { fumarate \&Emtricitabine }\end{array}$ & $\mathrm{D}_{1}$ & $224.38 \& 306.88 \mathrm{~nm}$ & $\begin{array}{l}3-21 \\
2-14\end{array}$ & 2011 & 86 \\
\hline $\begin{array}{l}\text { Simvastatin \& } \\
\text { Ezetimide }\end{array}$ & $\begin{array}{l}\mathrm{D}_{1} \\
\mathrm{D}_{1}\end{array}$ & $\begin{array}{l}219 \mathrm{~nm} \\
265 \mathrm{~nm}\end{array}$ & $\begin{array}{l}2-40 \\
1-20\end{array}$ & 2010 & 2 \\
\hline $\begin{array}{c}\text { Clopidogrel Bisulphate \& } \\
\text { Aspirin }\end{array}$ & $\mathrm{D}_{2}$ & $\begin{array}{l}254 \mathrm{~nm} \\
216 \mathrm{~nm}\end{array}$ & $5-30$ & 2010 & 2 \\
\hline $\begin{array}{l}\text { Atorvastatin calcium \& } \\
\text { Ezetimibe }\end{array}$ & $\mathrm{D}_{1}$ & $\begin{array}{l}266.6 \mathrm{~nm} \\
262.2 \mathrm{~nm}\end{array}$ & $3-15$ & 2010 & 87 \\
\hline $\begin{array}{l}\text { Strychnine \& } \\
\text { Brucine }\end{array}$ & $\begin{array}{l}D_{1} \\
D_{1}\end{array}$ & $\begin{array}{l}265.4 \mathrm{~nm} \\
256.4 \mathrm{~nm}\end{array}$ & $10-50$ & 2010 & 88 \\
\hline $\begin{array}{c}\text { Pantoprazole sodium \& } \\
\text { Itopride }\end{array}$ & $\mathrm{D}_{1}$ & $238.5-288 \mathrm{~nm}$ & $\begin{array}{l}3-15 \\
2-38\end{array}$ & 2010 & 89 \\
\hline
\end{tabular}




\begin{tabular}{|c|c|c|c|c|c|}
\hline $\begin{array}{c}\text { Drotaverine HCL \& } \\
\text { Paracetamol }\end{array}$ & $\mathrm{D}_{1}$ & $\begin{array}{l}303.5 \mathrm{~nm} \\
243.5 \mathrm{~nm}\end{array}$ & $\begin{array}{l}5-50 \\
5-60\end{array}$ & 2010 & 90 \\
\hline $\begin{array}{l}\text { Triprolidine } \mathrm{HCl} \& \\
\text { Pseudoephedrine } \mathrm{HCl}\end{array}$ & $\begin{array}{l}\mathrm{D}_{2} \\
\mathrm{D}_{2}\end{array}$ & $\begin{array}{l}321 \mathrm{~nm} \\
271 \mathrm{~nm}\end{array}$ & $\begin{array}{c}200-100 \\
10-50\end{array}$ & 2009 & 2 \\
\hline $\begin{array}{l}\text { Amoxicillin \& } \\
\text { Cephalexin }\end{array}$ & $\begin{array}{l}\mathrm{D}_{1} \\
\mathrm{D}_{2} \\
\mathrm{D}_{1}\end{array}$ & $\begin{array}{l}226 \mathrm{~nm} \\
274 \mathrm{~nm} \\
212 \mathrm{~nm}\end{array}$ & $10-60$ & 2009 & 91 \\
\hline $\begin{array}{l}\text { Cephalothin \& } \\
\text { Cefoxitin }\end{array}$ & $\mathrm{D}_{1}$ & $\begin{array}{c}235 \mathrm{~nm} \\
236.7 \mathrm{~nm}\end{array}$ & $4-32$ & 2009 & 92 \\
\hline $\begin{array}{l}\text { Tramadol \& } \\
\text { Ibuprofen }\end{array}$ & $\mathrm{D}_{1}$ & $\begin{array}{l}230.5 \mathrm{~nm} \\
280 \mathrm{~nm}\end{array}$ & $5-50$ & 2008 & 2 \\
\hline $\begin{array}{l}\text { Alendronate Na salt, } \\
\text { Clodronate disodium salt \& } \\
\text { Etidronate disodium salt }\end{array}$ & $\begin{array}{l}\mathrm{D}_{1} \\
\mathrm{D}_{2} \\
\mathrm{D}_{3} \\
\mathrm{D}_{1} \\
\mathrm{D}_{2} \\
\mathrm{D}_{3} \\
\mathrm{D}_{1} \\
\mathrm{D}_{2} \\
\mathrm{D}_{3}\end{array}$ & $\begin{array}{l}233 \mathrm{~nm} \\
245 \mathrm{~nm} \\
254 \mathrm{~nm} \\
236 \mathrm{~nm} \\
261 \mathrm{~nm} \\
284 \mathrm{~nm} \\
232 \mathrm{~nm} \\
243 \mathrm{~nm} \\
253 \mathrm{~nm}\end{array}$ & $25-600$ & 2008 & 93 \\
\hline $\begin{array}{l}\text { Doxylamine succinate, } \\
\text { Pyridoxine } \mathrm{HCl} \& \\
\text { Folic acid }\end{array}$ & $\mathrm{D}_{1}$ & $\begin{array}{c}270 \mathrm{~nm} \\
332.8 \mathrm{~nm} \\
309.2 \mathrm{~nm}\end{array}$ & $\begin{array}{c}2.5-50 \\
1-40 \\
1-30\end{array}$ & 2008 & 94 \\
\hline $\begin{array}{c}\text { Nebivolol \& } \\
\text { Hydrochlorothiazide }\end{array}$ & $\mathrm{D}_{1}$ & $\begin{array}{l}294.6 \mathrm{~nm} \\
334.6 \mathrm{~nm}\end{array}$ & $\begin{array}{c}8-40 \\
10-60\end{array}$ & 2008 & 95 \\
\hline $\begin{array}{l}\text { Metoprolol \& } \\
\text { Felodipine }\end{array}$ & $\mathrm{D}_{1}$ & $\begin{array}{l}222 \mathrm{~nm} \\
235 \mathrm{~nm}\end{array}$ & $\begin{array}{c}20-150 \\
10-60\end{array}$ & 2007 & 96 \\
\hline $\begin{array}{l}\text { Ranitidine } \mathrm{HCl} \& \\
\text { Ondansetron } \mathrm{HCl}\end{array}$ & $\mathrm{D}_{1}$ & $\begin{array}{l}340.8 \mathrm{~nm} \\
276.0 \mathrm{~nm}\end{array}$ & $\begin{array}{c}5-500 \\
2-30\end{array}$ & 2007 & 97 \\
\hline $\begin{array}{c}\text { Ondansetron \& } \\
\text { Paracetamol }\end{array}$ & $\mathrm{D}_{1}$ & $\begin{array}{l}302 \mathrm{~nm} \\
246 \mathrm{~nm}\end{array}$ & $\begin{array}{c}0.5-0.20 \\
20-30\end{array}$ & 2006 & 98 \\
\hline $\begin{array}{c}\text { Metoprolol \& } \\
\text { Hydrochlorothiazide }\end{array}$ & $\begin{array}{l}\mathrm{D}_{3} \\
\mathrm{D}_{1}\end{array}$ & $\begin{array}{l}281 \mathrm{~nm} \\
282 \mathrm{~nm}\end{array}$ & $\begin{array}{c}100-300 \\
12.5-37.5\end{array}$ & 2006 & 99 \\
\hline $\begin{array}{l}\text { Chlorprothixene \& } \\
\text { Amitryptyline }\end{array}$ & $\begin{array}{l}\mathrm{D}_{1} \\
\mathrm{D}_{2}\end{array}$ & $\begin{array}{c}316 \mathrm{~nm} \\
261.4 \mathrm{~nm} \& 268 \mathrm{~nm}\end{array}$ & $\begin{array}{l}0.5-50 \\
0.5-75 \\
\end{array}$ & 2005 & 100 \\
\hline $\begin{array}{l}\text { Phenytoin, } \\
\text { Barbital \& } \\
\text { Caffeine }\end{array}$ & $\mathrm{D}_{1}$ & $\begin{array}{l}207 \mathrm{~nm} \\
210 \mathrm{~nm} \\
230 \mathrm{~nm}\end{array}$ & $\begin{array}{c}0.24 \\
0.01-27 \\
0.049-27\end{array}$ & 2005 & 101 \\
\hline
\end{tabular}

Table 3: Determination of compounds in pharmaceutical sample along with AUC.

\begin{tabular}{|c|c|c|c|c|c|c|}
\hline Drug & $\begin{array}{c}\text { Order of } \\
\text { derivative }\end{array}$ & Wavelength (nm) & Linearity $(\mu \mathrm{g} / \mathrm{ml})$ & AUC & Year & Reference \\
\hline Tinidazole & $\mathrm{D}_{1}$ & $268 \mathrm{~nm}$ & $5-25$ & $314 \mathrm{~nm}-322 \mathrm{~nm}$ & 2015 & 102 \\
\hline Ofloxacin & $\mathrm{D}_{1}$ & $334 \mathrm{~nm}$ & $2-12$ & $284 \mathrm{~nm}-292 \mathrm{~nm}$ & 2015 & 103 \\
\hline Azelnidipine & $\mathrm{D}_{1}$ & $242.6 \mathrm{~nm}$ & $1-20$ & $250.5 \mathrm{~nm}-258.8 \mathrm{~nm}$ & 2015 & 104 \\
\hline Finofibric acid & $\mathrm{D}_{1}$ & $299 \mathrm{~nm}$ & $5-30$ & $275 \mathrm{~nm}-316 \mathrm{~nm}$ & 2015 & 105 \\
\hline Fluoxetine $\mathrm{HCl}$ & $\mathrm{D}_{0}$ & $226 \mathrm{~nm}$ & $5-25$ & $220 \mathrm{~nm}-231 \mathrm{~nm}$ & 2015 & 106 \\
\hline Ondasatron $\mathrm{HCl}$ & $\mathrm{D}_{2}$ & - & $2-10$ & $248 \mathrm{~nm}-254 \mathrm{~nm}$ & 2015 & 107 \\
\hline Ciprofloxacin $\mathrm{HCl}$ & $\mathrm{D}_{2}$ & _- & $2-10$ & $270 \mathrm{~nm}-278 \mathrm{~nm}$ & 2015 & 108 \\
\hline Ranitidine & $\mathrm{D}_{2}$ & $238 \mathrm{~nm}$ & $3-18$ & $310 \mathrm{~nm}-324 \mathrm{~nm}$ & 2015 & 109 \\
\hline Diclazuriline & $\mathrm{D}_{1}$ & $260 \mathrm{~nm}$ & $2-22$ & $300 \mathrm{~nm}-273 \mathrm{~nm}$ & 2014 & 110 \\
\hline Tadalafil & $\mathrm{D}_{1}$ & $297 \mathrm{~nm}$ & $5-50$ & $290 \mathrm{~nm}-304 \mathrm{~nm}$ & 2014 & 111 \\
\hline Carvedilol HCl & $\mathrm{D}_{1}$ & $233.7 \mathrm{~nm}$ & $1-14$ & $240 \mathrm{~nm}-244 \mathrm{~nm}$ & 2014 & 112 \\
\hline Rosuvastatin & $\mathrm{D}_{1}$ & $252 \mathrm{~nm}$ & $5-35$ & $247 \mathrm{~nm}-257 \mathrm{~nm}$ & 2014 & 113 \\
\hline Aceclofenac & $\begin{array}{l}\mathrm{D}_{0} \\
\mathrm{D}_{1}\end{array}$ & $\begin{array}{l}274.65 \mathrm{~nm} \\
259 \mathrm{~nm}\end{array}$ & $5-30$ & $269 \mathrm{~nm}-279 \mathrm{~nm}$ & 2014 & 114 \\
\hline Rupatadine fumarate & $\mathrm{D}_{1}$ & $214 \mathrm{~nm}$ & $1-30$ & $244 \mathrm{~nm}-255 \mathrm{~nm}$ & 2014 & 115 \\
\hline
\end{tabular}




\begin{tabular}{|c|c|c|c|c|c|c|}
\hline Imatimib mesylate & $\mathrm{D}_{1}$ & $\begin{array}{l}285 \mathrm{~nm}(\text { maxima }) \\
227 \mathrm{~nm}(\text { minima })\end{array}$ & $5-30$ & $237 \mathrm{~nm}-277 \mathrm{~nm}$ & 2013 & 116 \\
\hline Oxolamine citrate & $\mathrm{D}_{1}$ & $229.2 \mathrm{~nm}$ & $1-14$ & $228.6 \mathrm{~nm}-246.4 \mathrm{~nm}$ & 2013 & 117 \\
\hline Darunavir & $\mathrm{D}_{1}$ & $248 \mathrm{~nm}$ & $2-24$ & $257 \mathrm{~nm}-267 \mathrm{~nm}$ & 2013 & 118 \\
\hline Paliperidone & $\mathrm{D}_{0}$ & $238 \mathrm{~nm}$ & $3-18$ & $232 \mathrm{~nm}-244 \mathrm{~nm}$ & 2013 & 119 \\
\hline $\begin{array}{c}\text { Isoniazide \& } \\
\text { Paraamino salicylicacid }\end{array}$ & $\mathrm{D}_{1}$ & $\begin{array}{l}243 \mathrm{~nm} \\
257 \mathrm{~nm}\end{array}$ & $2-10$ & $258 \mathrm{~nm}-268 \mathrm{~nm}$ & 2013 & 120 \\
\hline Glipizide & $\mathrm{D}_{1}$ & $\begin{array}{l}\text { 286nm(maxima }) \\
263 \mathrm{~nm}(\text { minima })\end{array}$ & $5-25$ & $255 \mathrm{~nm}-295 \mathrm{~nm}$ & 2012 & 121 \\
\hline Zolpidem tartrate & $\begin{array}{l}\mathrm{D}_{0} \\
\mathrm{D}_{1}\end{array}$ & $\begin{array}{l}305 \mathrm{~nm}(\operatorname{maxima}) \\
263 \mathrm{~nm}(\text { minima })\end{array}$ & $5-50$ & $316 \mathrm{~nm}-263 \mathrm{~nm}$ & 2012 & 122 \\
\hline
\end{tabular}

\section{REFERENCES}

1. F. Rojas, C. Ojeda, J. Pavon, Talanta. 35, 753-761, 1988

2. J. Karpinska. Basic Principles and Analytical Application of Derivative Spectrophotometry, Jamal Uddin, 2012.

3. A. Owen. Uses of Derivative Spectroscopy, UV-Visible Spectroscopy, Application Note, Agilent Technologies, 1995.

4. D. Cameron, D. Moffatt. Apl Spec. 41, 539-44, 1987.

5. S. Kus, Z. Marczenko, N. Obarski. Chem Anal. 41, 899-927, 1996.

6. T. Owen. Fundamentals of Modern UV-Visible Spectroscopy: A Primer: Hewlett-Packard, 1996.

7. S. Upstone. Ultraviolet/Visible Light Absorption Spectrophotometry in Clinical Chemistry, Encyclopedia of Analytical Chemistry, 2000.

8. H. Willard, L. Merritt, J. Dean, F. Settle. Instrumental Methods of Analysis.

9. L. Mendham, R. Denney, J. Barnes, M. Thomas, B. Shivasankar. Vogel's Textbook of Quantitative Chemical Analaysis,

10. Beckett AH, Stenlake JB. Practical Pharmaceutical Chemistry.

11. Jeffery GH, Bassett J, Mendham J, Denney RC. Textbook of Quantitative Chemical Analysis.

12. M. Koba, K. Koba, L. Przyborowski. Acta Pol Pharm. 65, 289-294, 2008.

13. P. Minkiewicz, B. Pliszka, J. Dziuba, J. Oszmiański. Coll. Czech. Chem. Comm. 69, 1443-1452, 2004.

14. V. Saakov, V. Drapkin, A. Krivchenko, E. Rozengart, Y. Bogachev, M. Knyazev et al. Sprin. Sci. Bus. Med. 2012.

15. J. Karpińska. Talanta. 64, 801-822, 2004

16. A. Nangare, K. Pawa, A. Shinde. Der Pharma. Let. 6, 143-150, 2014,

17. A. Deosarkar, S. Deshpande, S. Walode. Amer. J. Pharm. Tech. Res. 2, 678-685, 2012.

18. S. Balaram, A. Ramesh, P. Jagadish. Res. J. Pharma. Tech. 7, 1113-1117, 2014

19. N. Abdullah, M. Hassan, R. Hassan. Ara. J. Chem. 2005

20. M. Ranganath, K. Reddy. Intern. J. Pharm. Tech. Res. 4, 123-126, 2010.

21. R. Chaudhari. Asa. J. Pharma. Clin. Res. 6, 162-164. 2013.

22. S. Baravaliya, J. Tandel, M. Maste, M. Mohite. Intern. J. Pharm. Tech. Res. 4, 2013.

23. C. Rajyalakshmi, T. Benjamin, C. Rambabu. Intern. J. Pharm. Tech. Res.5, 217-221, 2013.

24. P. Dhanawade, R. Kane. Inter. J. Res. Pharma. Biom. Sci. 3, 1300-1305, 2012.

25. P. Jain, H. Jivani, R. Khatal. S. Surana. Ind. J. Pharm. Sci. 74, 168, 2012.

26. H. El Lakiss, M. Ilie, D. Baconi, D. Bălălău. Farmacia. 60, 565-570, 2012.

27. V. Panditi, A. Vinukonda. Inter. J. Chem. Tech. Res. 3,18-22, 2011.

28. R. Pandey, P. Patil, M. Patil, P. Deshmukh, S. Bari. Pharma. Meth. 3, 4-8, 2012.

29. B. Szaniszlo, C. Iuga, M. Bojita. Farmacie. 84, 398-401, 2011.

30. S. Pandey, P. Pandey, S. Dubey, U. Chaturvedi, A. Rai. Tha. J Pharma. Sci. 36, 2012

31. S. Malipatil, M. Deepthi, S. Patil, K. Jahan. Int. J. Pharm. Pharm. Sci. 3, 13-15, 2011.

32. A. Sokol, J. Karpinska, R. Telecka, B. Starczewska. Acta. Pol. Pharma. 68, 169-177, 2011.

33. K. Chiranjeevi, K. Channabasavaraj, P. Reddy, P. Nagaraju. Intern. J. Chem. Tech. Res.; 3, 58-62, 2011.
34. S. Sharma, M. Sharma. Eur. J. Appl. Sci. 3, 81-85, 2011.

35. E. Hassan, M. Hagga, H. Al Johar. J. Pharm. Biom. Ana. 24, 659-665, 2001

36. M. Game, D. Sakarkar, K. Gabhane, K. Tapar. Intern. J. Chem. Tech. Res. 2(2), 1259-1262, 2010

37. D. Madhuri, K. Chandrasekhar, N. Devanna, G. Somasekhar. Intern. J. Pharma. Sci. and Res. 1(4), 222-231, 2010.

38. S. Acharjya, P. Mallick, P. Panda, K. Kumar, M. Annapurna. J. Adv. Pharma. Tech. Res. 1, 348, 2010.

39. O. Lastra, I. Lemus, H. Sánchez, R. Pérez. J. Pharma. Bio. Ana. 33, 175$180,2003$.

40. H. Thakkar, K. Patel. Chron. Y. Sci. 1(3), 22, 2010.

41. M. Sali, A. Barhate, V. Patil, A. Bhadoriya, V. Choudhari, B. Kuchekar et al.

Der. Pharm. Che. 2, 12-16, 2010.

42. B. Yilmaz. Int. J. Pharma. Bio. Sci. 1(1), 1-15, 2010.

43. V. Shirvi. . J. Adv. Pharma. Tech. Res. 1, 548, 2010.

44. A. Charoo, M. Bashir, E. Abdalla, K. Ali. Ana. let. 42, 2232-2243, 2009.

45. J. Krzek, H. Woltyńska, U. Hubicka, Ana. Let. 42, 473-482, 2009.

46. S. Ulu, F. Elmal. Ana. Let. 42, 2254-2270, 2009.

47. B. Stanisz, S. Paszun, M. Lesniak. Acta. Pol. Pharma. D. Res. 66(4), $343-$ 349, 2009.

48. M. Sharma, D. Mhaske, M. Mahadik, S. Kadam, S. Dhaneshwar. Ind. J. Pharma. Sci. 70, 258, 2008.

49. P. Anumolu, S. Gurrala, V. Yeradesi, S. Puvvadi, S. Chavali. Trop. J. Pharma. Res. 12(2), 227-232, 2013.

50. M. Himaja, J. Kalpana, C. Anbarasu. Int. J. Pharm. Pharm. Sci. 6, 302304, 2014

51. N. Shreef. Raf. J. Sci. 19, 38-46, 2008

52. J. Millership, F. McCaffrey, D. Tierney. J. Pharma. Bio. Ana. 48(2), 408413, 2008

53. U. Kemal, P. Murat, K. Elif, O. Fyyaz. Turk. J. Pharma. Sci. 5, 1-16, 2008 .

54. M. Ansari, M. Kazemipour, M. Baradaran, H. Jalalizadeh. Ira. J. P'cology. Therap. 3(1), 21-25, 2004

55. K. Gunjan, V. Deshmukh, P. Kashya, R. Bawankar, Y. Vaishnav, D. Biswas. J. Pharm. Pharm. Sci. 6, 102-104, 2010.

56. Z. Aydoğmuş, E. Yılmaz, S. Yörüsün, S. Akpınar. Intern. Sch. Res. N. 2015.

57. A. El-Zinati, M. Abdel-Latif. The O. Ana. Chem. J. 8(1), 2015.

58. E. Souri, A. Rahimi, N. Ravari, M. Tehrani. Iran. J. Pharma. Res. 14, $435,2015$.

59. V. Hoang, N. Hue, N. Tho, H. Nguyen. Spec. Acta. P. A: Mol. Biom. Spec. 139: 20-27, 2015.

60. M. Stolarczyk, A. Maślanka, A. Apola, W. Rybak, J. Krzek. Spec. Acta. P. A: Mol. Biom. Spec. 148, 66-71, 2015

61. K. Thula, D. Patel, D. Maheshwari. Intern. J. Pharma. Sci. 31, 243-247, 2015.

62. V. Hoang, D. Ly, N. Tho, T. Minh, H. Nguyen. The Sci. W. J. 2014.

63. C. Mathew, B. Suman, M. Ajitha, P. Babu. Ori. J. Che. 30, 1385-1389, 2014

64. R. Sversut, I. Alcântara, A. Rosa, A. Baroni, P. Rodrigues, A. Singh, M. Amaral, N. Kassab et al. Arab J. Che. 2014.

65. E. Pandya, P. Kapupara, K. Shah. J. Chem. Pharma. Res.6, 912-924, 2014

66. B. Patel, A. Jadav, H. Solanki, S. Parmar, V. Parmar, A. Captain. Intern. 
J. Pharma. Res. Rev. 2, 1-6, 2013.

67. A. Jadav, B. Gohel, S. Mital, B. Patel, S. Parmar. Int. J. Pharma. Tech Res. 5, 1155-1160, 2013.

68. P. Joshi, S. Parmar, B. Patel, Intern. J. Spectro. 2013.

69. S. Gulve, K. Tarkase, D. Mundhe, P. Hajare. Int. J. Pharma. Tech. Res; 5, 122-127, 2013.

70. V. Deshmukh, D. Wagh, S. Vassa, K. Gujar. Intern. Res. J. Pharma. 4,115-119, 2013

71. R. Rele, S. Sawant, S. Patil. Intern. Res. J. Pharma. 4, 43-48, 2013.

72. G. Abirami, T. Vetrichelvan. Int. J. Pharm. Pharm. Sci. 5, 488-92, 2013.

73. P. Pawar, S. Hapse, M. Salve, A. Agarkar. Intern. J. D. Res. Tec. 3, 3744, 2013.

74. P. Anumolu. Intern. Res. J. Pharma. 4,215-219, 2013.

75. M. Attimarad, B. Al-Dhubiab, I. Alhaider, A. Nair. Chem. Cen. J. 6,1, 2012.

76. D. Patel, R. Shah, A. Patel, K. Ponal. Intern. J. Pharma. Sci. 3, 2012

77. P. Patel, B. Marolia, S. Shah, D. Shah. Intern. Res. J. Pharm. 3, 259-62, 2012

78. A. Shetty, R. Sireesha, M. Ahmed. Intern. J. Pharm. Tech. Res.4, 1676$1685,2012$.

79. P. Mohite, R. Pandhare, S. Khanage. Adv. Pharma. Bull. 2, 115, 2012.

80. S. Mujbaile, P. Prasad, S. Wate. J. Pharm. Biol. Sci. 4, 5-8, 2012.

81. A. Sutar, A. Battewar, A. Hable, V. Modak, V. Choudhari, C. Magdum. Adv. Pharma. Bull. 2008.

82. A. Chabukswar, S. Tambe, V. Choudhari, S. Sharma, M. Mohokar, S. Chate. Res. J. Pharma. Tech. 5, 950-4, 2012.

83. S. Gondane, M. Deshpande, M. Mahajan, S. Sawant. Intern. J. Chem Tech. Res. 3, 620-624, 2011.

84. S. Kumbhar, S. Jadhav, N. Bhatia, M. Bhatia. Int. J. Pharm. Pharm. Sci. 3, 195-7, 2011.

85. S. Wankhede, A. Mahajan, S. Chitlange. Der. Pharma. Chemica. 3, 269273, 2011.

86. V. Choudhari, S. Ingale, S. Gite, D. Tajane, V. Modak, A. Ambekar et. al. Pharma. Met.; 2,47-52, 2011

87. Y. Baghdady, M. Al-Ghobashy, A. Abdel-Aleem, S. Weshahy. Intern. J. Chem. Stu. 1, 2013.

88. B. Ganesan, P. Perumal, V. Manickam, S. Srikakolapu, S. Gotteti, L. Thirumurthy. Int. J. Pharm. Tech. Res. 2, 1528-1532, 2012.

89. D. Bageshwar, A. Pawar, V. Khanvilkar, V. Kadam. Asi. J. Pharm. Clin. Res. 3,221-3, 2010.

90. S. Mahaparale, R. Telekone, R. Raut, S. Damle, P. Kasture. Ind. J. Pharma. Sci. 72,133, 2010

91. S. Dikran, M. Hussan. Der. Pharma. Chem. 6, 169-173, 2011.

92. J. Murillo, J. Lernus, L. Garcia. J. Pharma. Bio. Ana. 14, 257-266, 1996

93. M. Koba, K. Koba, L. Przyborowski. Acta. Pol. Pharm. 65, 289-294, 2008.

94. A. Pathak, S. Rajput. Ind. J. Pharma. Sci. 70, 513, 2008.

95. D. Shah, K. Bhatt, R. Mehta, S. Baldania. J. AOAC Interna. 91,10751082,2008

96. H. Salem, O. Abdallah. Am. J. App. Sci. 4, 709-717, 2007.

97. S. Pillai, I. Singhvi. Ind. J. Pharma. Sci. 69, 2007.

98. P. Kumar, M. Krishna, P. Prakash, B. Kumar, P. Madhusudhan. J. Chem. 3, 134-136, 2006.

99. M. Stolarczyk, R. Ekiert, J. Krzek, W. Rzeszutko. Acta. Pol. Pharma. $63,169-173,2006$

100. J. Karpińska, J. Szostak, Spec. Act. Part A: Mol. Bio. Spec. 61, 975-981, 2005.

101. A. Abbaspour, R. Mirzajani. J. Pharma. Bio. Ana. 38, 420-427, 2005.

102. S. Masthannamma, S. Ananta, B. Naik, A. Kumar. Intern. J. Res. Deve. Pharm. L. Sci. 1582-1589, 2015.

103. S. Masthannamma, S. Ananta, B. Naik, A. Kumar. Ind. J. Pharma. Sci. 92,333, 2015.

104. R. Rele. J. Res. Deve. Pharm. L. Sci. 1624-1629, 2015.

105. A. Alageswaran, S. Jerad, V. Niraimathi. Acta. Pol. Pharma. 95,269-273, 2015.

106. M. Annapurna, D. Pradhan. Chem. Sci. Trans. 1, 697-701.

107. S. Jadhav, R. Kharat, A. Tamboli. Pharma. Tutor. 3, 42-46, 2015.

108. R. Kharat, S. Jadhav, D. Tamboli, A. Tamboli. Intern. J. Adv. Sci. Res. $1,137-144,2015$

109. A. Mali. Intern. J. Ana. Pharma. Bio. Sci. 4,7-16, 2015

110. T. Ibrahim. Validated UV-spectrophotometric estimation of diclazurilin API and drug product. Chem. Sci. Trans 2, 710-71, 2006.

111. Z. Khan, A. Patil, A. Shirkhedkar. Intern. J. Spec. 2014.
112. R. Rele, P. Tiwatane. Res. J. Pharma. Tech. 7, 1459-1462, 2014.

113. R. Lahare, A. Phuge, A. Gite, A. Jadhav. Int J. P. Appl. Bio. Sci. 6, 16974, 2014.

114. C. Srujani, B. Sravanthi, D. Madhuri. Sch. Aca. J. Pharm. 3, 471-476, 2014.

115. R. Rele, P. Tiwatane. Asi. J. Res. Chem. 7, 2014.

116. S. Patil, R. Doijad. Asi J. Pharma. Cli. Res. 6, 54-57, 2013.

117. V. Rajan. Res. J. Pharm. Tech. 7, 1150-1152, 2014.

118. S. Vanukuri, S. Mastanamma, G. Alekhya. Intern J. Pharm. Pharma. Sci. 6, 2013.

119. S. Kancharla, G. Pavan, N. Paladugu, S. Bonthu, D. Poloju. Int. J. Bio. Pharm. Res. 4, 412-416, 2013.

120. S. Vijayaraj. Asi. J. Pharm. Clin, 6, 108-110. 2013.

121. D. Rathod, M. Dole, S. Sawant. Asi. J. Pharm. Clin. Res. 5, 102-104, 2012.

122. M. Mahajan, S. Sawant. Int. J. Chem. Tech. Res. 4, 403-408, 2012

123. S. Acharjya, M. Rao, B. Kumar, M. Annapurna. J. Adv. Sci. Res. 1, 4247, 2011.

124. S. Patel. 2011

125. S. Gujarathi, A. Shah, S. Jagdale, P. Datar, V. Choudhari, B. Kuchekar. Int. J. Pharma. Sci. Rev. Res. 3, 115-119, 2010

126. K. Shah, T. Desai, A. Behera, R. Pal, K. Girhepunje. Res. J. Pharma. Bio. Chem. Sci. 2010.

127. K. Gupta, V. Samrit, V. Thakur, A. Hemke. J. Chem. Pharm. Res. 2, 467472, 2010.

128. S. Acharjya, P. Mallick, P. Panda, K. Kumar, M. Annapurna. J. Adv. Pharma. Tech. Res. 1, 348, 2010.

129. Y. Prasad, K. Rajasekhar, V. Shankarananth, H. Yaminikrishna, S Saikumar, P. Reddy. J. Pharma. Res. 2, 1593, 2009. 